\title{
METHODS FOR COMPARING SERVO GRIPPERS FOR MINI AND MICRO ASSEMBLY APPLICATIONS
}

\author{
Ilpo Karjalainen and Reijo Tuokko \\ Tampere University of Technology, Institute of Production Engineering, Robotics and \\ Automation Laboratory, P.O Box 58933101 Tampere, Finland. Tel. +358-3-3115 2111, Fax. \\ +358-3-31152753,Email: ilpo.karjalainen@tut.fi and reijo.tuokko@tut.fi
}

Abstract: In the future the assembled parts become smaller and the precision demands increase in many cases. Grippers are one factor of the solution to make a competitive automation assembly system. Grippers have to be flexible and also capable of holding parts without dropping them during accelerations. Other demands for a gripper for mini and micro assembly applications are, among other things, accuracy and good force/size ratio. By using servo grippers also in high-precision applications for miniature-size parts, a noticeably amount of time will be saved. This paper presents two different kinds of developed two-fingered servo grippers with parallel moving mechanics, as well as the developed test systems for servo grippers and some test results.

Key words: Servo gripper, micro assembly

\section{INTRODUCTION}

In the future the assembled parts become smaller and the precision demands increase in many cases. Grippers are one factor of the solution to make a competitive automation assembly system. Micro gripper research has been widely made in last twenty years $[1,2]$. So far most commercially available grippers are two-fingered or at most three-fingered [3]. Demands for a gripper for mini and micro assembly applications are, among other things, accuracy and good force/size ratio. By using servo grippers also in 
high-precision applications for miniature-size parts, a noticeably amount of time will be saved. In most of the servo gripper applications any kind of angular movement of fingers is a problem. In reference [4] weakness of the rotational movements of gripping arms is mentioned and analyzed. This paper presents two different kinds of developed two-fingered servo grippers with parallel moving mechanics, as well as the developed test systems for servo grippers and test results of servo grippers.

\section{DEVELOPED SERVO GRIPPERS}

In this work the target was to develop a high-precision parallel servo gripper with non-synchronous driven fingers based on piezoelectric linear motors. Target for the gripper was that the structure is simple and the gripper does not drop parts under high accelerations. Mechanically the gripper is based on two standing wave ultrasonic motors (UM), two high precision linear guides and two linear encoders (resolution 0.2 micrometer). The driving stroke each of the individual fingers is $5 \mathrm{~mm}$ and the maximum opening is $10 \mathrm{~mm}$. Weight of the servo gripper is less than 300 grams. The gripper is mechanically designed to be compact and volumetrically small. Volumetrical size is $89 \mathrm{~mm} \times 78 \mathrm{~mm} \times 26.5 \mathrm{~mm}$ without fingers. The gripper fingers can be freely programmable and servo driven individually to the defined points with exact velocity sequence confirmed via controller's HMI. A camera or a microscope can be easily integrated to the gripper through a hole in the center of the gripper. This solution enables continuous monitoring of the parts during grasping, handling and releasing. Gripper has PC-control including HMI and joystick for driving finger and teaching cycle points. Figure 1 shows a view of the ultrasonic motor-based gripper.

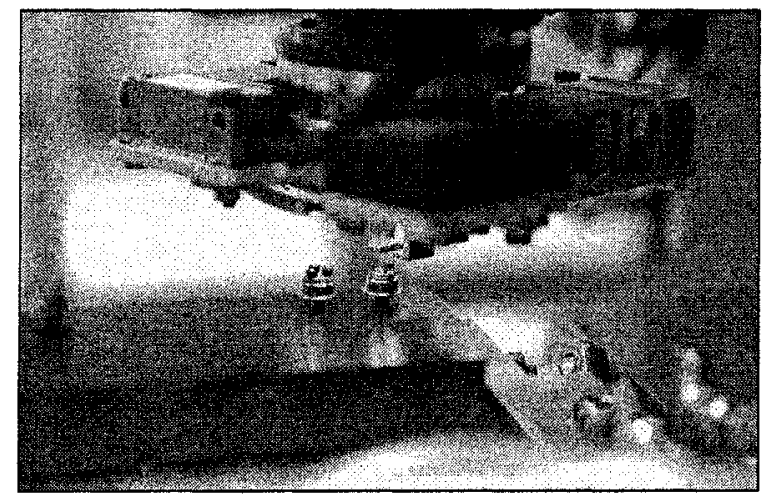

Figure 1. A servo gripper based on PZT motors in assembly work. 
A state-of-the-art servo gripper based on ball screws and rotating motors (RM) was built to research this traditional technology also in high-precision applications, see figure 2 . The developed gripper is a two-fingered servo gripper with position feedback including the following main components: two small DC-motors with brushes; two high-precision, preloaded ball screws; two high-precision linear guides and two high-resolution encoders (resolution 0.2 micron). This gripper motors has mostly same quantities than the gripper based on ultrasonic motors, like e.g. HMI interface. Fingers are driven without gears changing rotating ratio between motor axes and ball screws. Motors are directly connected to the ball screws with mechanical coupling.

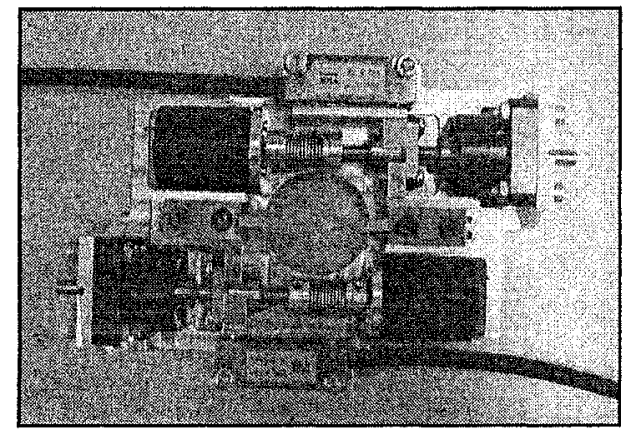

Figure 2. The developed gripper based on rotating DC-motors.

\section{TESTING METHODS FOR SERVO GRIPPERS}

Basically, servo grippers have requirements similar to those of normal grippers. When comparing servo grippers, it is expedient to concentrate on qualities that are characteristics of servo grippers and that are most important for normal on/off grippers.

Motion-controlled servo grippers are able to carry out movements, which are shorter than the maximum stroke. This measuring problem can be divided to two individual parts: first, measuring linearity, accuracy and repeatability of the movement, and, second measure position and velocity responses of fingers. In this project the accuracy and repeatability of the gripper fingers is measured with a laser interferometer [5] and a plane mirror mounted to a finger axis. A small plane mirror is attached to the gripper fingers one finger at a time. ISO 230-2 standard determines accuracy and repeatability tests of positioning numerically controlled axes [6]. There are several possibilities for measuring positioning and velocity responses of the gripper fingers. In 
research environments e.g. a laser interferometer with high sampling rate can be used.

A servo gripper's finger-axis can became unstable when undergoing transporting accelerations, due to possible instability of the finger-axis. In industrial applications dropping of the parts during handling is a considerable problem. When parts become smaller and accelerations higher the problem is even bigger. In servo gripper research it is especially important to test the behaviour of the gripper under specific accelerations. Some gripper actuators such as moving coil linear motors or pneumatic servo valves can become unstable even under relatively low accelerations. In Tampere University of Technology a Cartesian robot has been developed $[7,8]$, which is suitable for researching and comparing different grasping and handling techniques, see figure 3 .

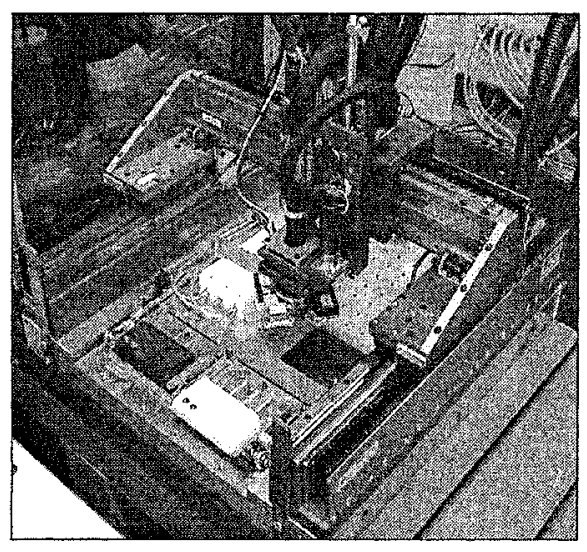

Figure 3. Servo gripper connected to Cartesian robot [7].

With the robot's microscope also fine positioning of parts can be researched. With this system the grippers can be tested under specified horizontal, vertical and rotational accelerations. With the same system one can study pick and place times in different assembly operations and processes. With the achieved results one can optimize force, size of the gripper and shape of the gripper's fingers.

A servo gripper's finger-axis can became unstable in a grasping situation, because of the grasping force. When force feedback is used in servo gripper, it is also possible to control grasping force. Practical tests have been made with grippers for different materials, control algorithms, and approach velocities. Fingers' grasping force was measured with a 6 DOF force sensor so the direction of each force can also be seen. The other finger at the same time had an opposing flange. 
Two-fingered servo grippers can be made in two different ways. Servo gripper's fingers can move parallel or individually. When fingers are moving individually the gripper is more flexible, but problems may arise with the centering of the part to be grasped. Depending on the centering of the part and the deviation in the axis forces the final positioning and the settling time may vary. We have researched centering behavior and settling times of grippers with two individually moving fingers by measuring positioning and velocity responses from both fingers during grasping. Fingers can also be driven synchronously so that one finger is moving to a closed position and the other to an open position. In other words, the gripper is able to move the part during grasping. A finger can also be driven individually, and nonsynchronously, and thereby increase flexibility in grasping situations. These qualities were tested with case products.

\section{TEST RESULTS FOR THE SERVO GRIPPERS}

Using the results of the introduced bidirectional measurement the repeatability (3s) of gripper fingers can be calculated with equation 1 , where $\mathrm{q}$ is the independent measurement result. The measurement was repeated five times with a stroke of $5 \mathrm{~mm}$.

$$
\pm 3 S_{q}= \pm 3 \sqrt{\frac{\sum_{k=1}^{N}\left(q_{k}-\bar{q}\right)^{2}}{N-1}}
$$

In table 1 the accuracy is presented; repeatability is presented as a worstcase repeatability.

Table 1. Laser measurements results of grippers axes (3o)

\begin{tabular}{lll}
\hline & Accuracy & Repeatability \\
\hline Finger 1 (rotating DC-motor) & 10,5 micron & $\pm 4,1$ micron \\
Finger 2 (rotating DC-motor) & 7,1 micron & $\pm 2,4$ micron \\
Finger 1 (ultrasonic motor) & 18,6 micron & $\pm 1,3$ micron \\
Finger 2 (ultrasonic motor) & 19,4 micron & $\pm 0,8$ micron \\
\hline
\end{tabular}

Accuracy, repeatability, velocity, and settling times were measured for both of the gripper's fingers. The main focus was on accuracy and repeatability tests of the fingers. Another objective was that the axes actual position be able to follow the command position as exactly as possible. 


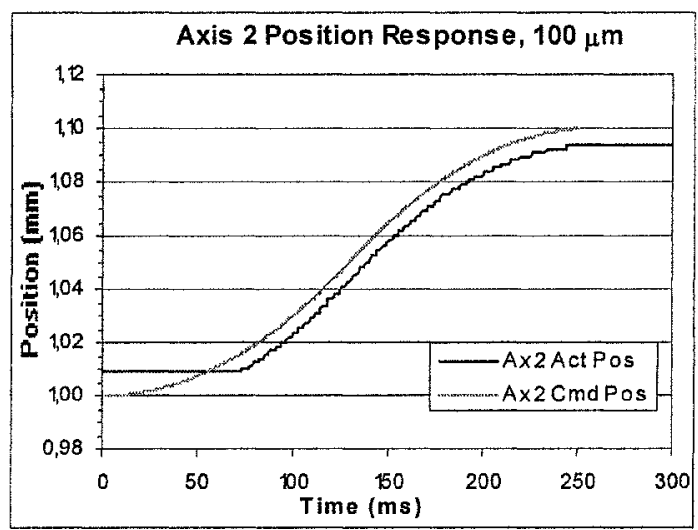

Figure 4. Position response of UM-finger in a 100 micron movement.

The actual position is not able to achieve command positioning with a PID controller, if the driven length is only 100 microns, see figure 4 . The problem is similar with both of the axes. The reason is a static friction of the linear guide, and a low force of the UM at low velocities. Increasing the velocity makes movement unstable, in short movements.

Position responses of rotating RM-gripper axis 100 micron movement is introduced in figure 5. Position error in the end of the movement is smaller than with ultrasonic motor axes.

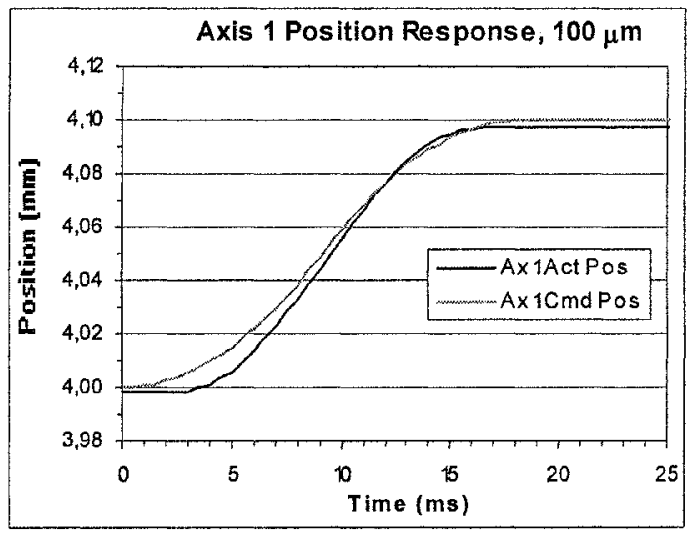

Figure 5. Position response of RM-finger in a 100 micron movement.

Behaviors of the developed grippers under accelerations were tested with the introduced research environment. The grippers did not drop parts under 
maximum accelerations available: $40 \mathrm{~m} / \mathrm{s}^{2}$ in perpendicular horizontal directions and $30 \mathrm{~m} / \mathrm{s}^{2}$ in vertical direction. The gripper based on ultrasonic motors was able to hold grasped parts under same accelerations also when the power of the ultrasonic motors was turned off. In this situation the grasp hold was maintained with the static friction between the motor's driving tip and the slide.

Grasping force depend on finger approaching velocity, which was 1 to 10 $\mathrm{mm} / \mathrm{s}$. Gripper based on ultrasonic motors, grasping force was 8 to 10 Newton. For a gripper based on DC-motors grasping force was little higher, between about 20 to 27 Newton.

Centering capability of the two independently moving fingers in grasping situation was tested. The graph in figure 6 shows the situation when finger 2 stays still and finger 1 starts to grasp the part. Graph shows that the positioning error of finger 2 increases to 18 micron and does not return to the original command position, but new position is stable.

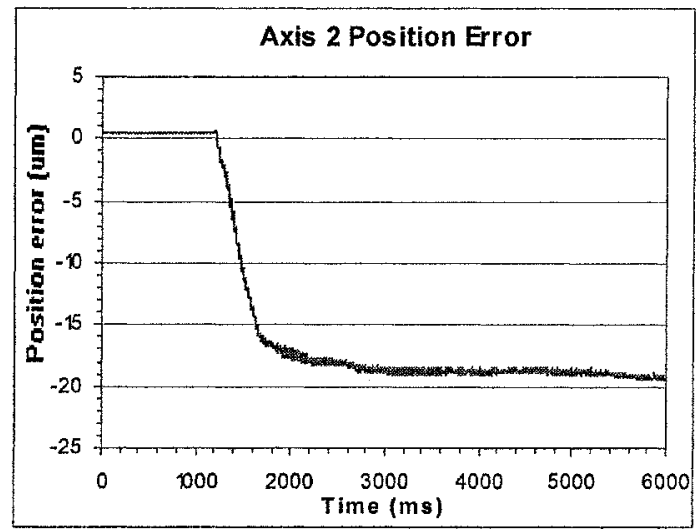

Figure 6. Position error of finger 2 in grasping

Centring behaviour with the gripper based on RM is better than with UM. The reason is lower friction. With RM, axes improve their centring after grasping, but it takes several seconds.

For testing of the gripper's quantities in practice a miniature size gearbox was assembled and disassembled. This gearbox consists of five different parts. This gearbox was used as a case product. Biggest part of the planetary gearbox is the tube, which diameter is 8 millimeter and smallest part is the planetary wheel, the diameter of which is 2,2 millimeter. In assembly process it was difficult to assemble some of the parts. According to our experiences it can be concluded that parallel mechanism is very important feature for a servo gripper when several different size parts are handled with same fingers. When parts are miniature-sized, this is even more important. 
Individually moving fingers increase flexibility in grasping situation and together with integrated microscope grasping is easy to do. With a traditional DC-motor, with ball screw technology, it is possible to make a high accuracy gripper within a few microns of repeatability, when all mechanical components are of high precision.

\section{CONCLUSION}

In this paper two different kinds of developed two-fingered servo grippers with parallel moving mechanism have been presented. Parallel mechanism is very important feature for a servo gripper when several different size parts are handled with same fingers. When parts are miniature size, this is even more important. Moving fingers individually increases flexibility in grasping situation. Proposals for tests and measurements for two-fingered servo gripper with parallel movement have been developed and introduced as well as some test results.

\section{REFERENCES}

1. M.A. Carrozza, A. Eisinberg, A. Menciassi, D. Campolo, S. Micera, P. Dario: "Towards a force-controlled microgripper for assembling biomedical microdevices", J. Micromech. Microeng. pp. 271-276 No. 10 (2000). Printed in the UK.

2. Y. Bellouard. "Microgrippers Technologies Overview", 1998 IEEE International Conference on Robotics and Automation, Workshop WS4: Precision Manipulation at Micro and Nano Scales, pp. 84 - 109, May 16 - 20.. Leuven, Belgium, 1998.

3. Wen-Han Qian, Hong Qiao, S.K. Tso: "Synthesizing Two-Fingered Grippers for Positioning and Identifying Objects, IEEE Transactions on Systems, Man, and Cybernetics-Part B: Cybernetics Vol. 31 No. 4, August 2001.

4. R. Keoschkerjan, H. Wurmus "A Novel Microgripper with Parallel Movement of Gripping Arms", Actuator 2002, 8th International Conference on New Actuators", pp. 321-324, Bremen, Germany, June 10-12 2002.

5. Anonym: "HP Laser Interferometer $5527 \mathrm{~A} / \mathrm{B}$ General Information", $50 \mathrm{p}$.

6. Anonym: "ISO standard 230-2", 1997, 15 p.

7. Heikkilä, R., Tuokko R. Development of a High Precision and High Performance Mini Robot - Technological Highlights and Experiences, International Precision Assembly Seminar, Bad Hofgastein, Austria, 17-19 March 2003, pp. 49-54.

8. I. Karjalainen, J. Uusitalo, T. Sandelin, R. Heikkilä, R. Tuokko: "Intelligent and Flexible End-Effector with Integrated Microscope - A Pilot Platform for Mini and Micro Assembly Research", INES 2001 International Conference on Intelligent Engineering Systems, 16-18 September 2001, Helsinki, Finland, pp. 307-311. 